\title{
Erratum
}

In the article "Effects of Olmesartan, an Angiotensin II Receptor Blocker, and Amlodipine, a Calcium Channel Blocker, on Cardio-Ankle Vascular Index (CAVI) in Type 2 Diabetic Patients with Hypertension" by Yoh Miyashita., which appeared in JAT 2009, 16: 621-626, are incorrect. The editiorial staff apologizes to the authors and all readres. The correct are presented with corresponding below.

\section{Effects of Olmesartan, an Angiotensin II Receptor Blocker, and Amlodipine, a Calcium Channel Blocker, on Cardio-Ankle Vascular Index (CAVI) in Type 2 Diabetic Patients with Hypertension}

False

\begin{abstract}
Methods: Seventy type 2 diabetes mellitus patients with hypertension were enrolled and randomly divided into two groups. One group was administered olmesartan medoxomil $10 \mathrm{mg} / \mathrm{day}$ for 12 months (ARB group), and the other group was administered amlodipine besilate 5 $\mathrm{mg}$ /day for 12 months (Ca blocker group).
\end{abstract}

\section{Subjects and Methods}

\section{Subjects}

A randomized, open study was performed. Seventy type 2 diabetes mellitus patients with hypertension, who attended Sakura Medical Center of Toho University as outpatients, were enrolled. Patients were excluded if they had received insulin therapy or had diabetic retinopathy, nephropathy and previous cardiovascular and cerebrovascular diseases. All patients had taken no antihypertensive drugs before this study. The enrolled subjects were randomly divided into two groups. One group was administered olmesartan 10 $\mathrm{mg} / \mathrm{day}$ for 12 months (ARB group, $n=35$ ), and the other group was administered amlodipine $5 \mathrm{mg} /$ day for 12 months (Ca blocker group, $n=35$ ). These drugs were taken in the morning after 12 hours of fasting. During this study, all patients maintained the same diet and exercise therapies, and did not change medications. All subjects received nutrition education from a dietitian every month. This study was approved by the institutional review board. The purpose of this study was explained to the subjects, and consent was obtained for participation in the study and also for release of the study data.
Correct

Methods: Seventy type 2 diabetes mellitus patients with hypertension were enrolled and randomly divided into two groups. One group was administered olmesartan medoxomil $20 \mathrm{mg} /$ day for 12 months (ARB group), and the other group was administered amlodipine besilate 5 $\mathrm{mg} /$ day for 12 months (Ca blocker group).

\section{Subjects and Methods}

Subjects

A randomized, open study was performed. Seventy type 2 diabetes mellitus patients with hypertension, who attended Sakura Medical Center of Toho University as outpatients, were enrolled. Patients were excluded if they had received insulin therapy or had diabetic retinopathy, nephropathy and previous cardiovascular and cerebrovascular diseases. All patients had taken no antihypertensive drugs before this study. The enrolled subjects were randomly divided into two groups. One group was administered olmesartan 20 $\mathrm{mg} / \mathrm{day}$ for 12 months (ARB group, $n=35$ ), and the other group was administered amlodipine $5 \mathrm{mg} /$ day for 12 months (Ca blocker group, $n=35$ ). These drugs were taken in the morning after 12 hours of fasting. During this study, all patients maintained the same diet and exercise therapies, and did not change medications. All subjects received nutrition education from a dietitian every month. This study was approved by the institutional review board. The purpose of this study was explained to the subjects, and consent was obtained for participation in the study and also for release of the study data. 